\title{
Change Detection in Satellite Images Based On Contourlet Image Fusion and RFLICM Clustering
}

\author{
Renjana.G, Kavitha.N.Nair, \\ $P G$ Scholar, Department of ECE, University College of Engineering, Muttom, Kerala - 685587 \\ Lecturer, Department of ECE University College of Engineering Muttom, Kerala - 685587
}

\begin{abstract}
Change detection of the satellite images based on the contourlet image fusion and reformulated fuzzy clustering for change detection. Here the image fusion is used to produce difference image from log ratio and mean ratio imagesFor an optimal difference image, it should retain the unchanged areas and enhance the changed areas. So contourlet based image fusion is proposed to generate the difference images. This difference image should preserve the curves so that the clarity of the image become more.The method of differencing images from the log ratio and mean ratio of the individual images. The difference image have better quality. To process the difference image is to discriminate changed regions from the unchanged regions using reformulated fuzzy local information c means algorithm which is insensitive to noise. Experiment result shows that this approach provide better performance than its preexistence. Verified the proposed fusion algorithm and clustering algorithm by well -known image fusion measures and calculate the percentage correct classification.On the basis of experimental results it was found that performances of the proposed fusion method and clustering methods are better than the preexisting one.
\end{abstract}

Keywords: Contoulettransform,Image fusion, Reformulated fuzzy clustering, change detection.

\section{INTRODUCTION}

Change detection is atechnique that identifying the changes by analyzing images obtained from the same geographical are at different time[1].The change detection of the regions ,multi temporal satellite images are used.Here the regions of same are at different periods of great interest.The process have many applications in different fields. The main application including in medical diagnosis[2],remote sensing,video surveillance.For choosing the images presence of multiplicative speckle noise.Change detection can be done in many ways including supervised or unsupervised manner.In the supervised technique a set of training patterns are required. It is of very much difficult.But in the case of unsupervised manner, there is no need of training data.So unsupervised techniques are better than supervised techniques.

This literature ,three main steps are adopted to perform unsupervised change detection,,1)Pre processing of images,2)Comparison of image by ratio merthod,3)Analysis of the difference image for change detection. The purpose of the step 1 include reduction of noise, geometric and radiometric correction and co registration.In the next step involved two images taken as the input and compered pixel by pixel to produce difference image.For producing the difference image ,ratio operator and subtraction operator are most commonly used.In the case of ratio operator,two pre processed images are taken as the input and apply pixel by pixel operator to it,there by changes are obtained.Generally using ratio operator instead of the differencing operator.Because differencing operator is affected by caliberationerrors[2].

After performing all,change detection is done on difference image.For this several methods are used,like sensitive or non sensitive method .Histogram thresholding is one kind in this threshold value may be detected by automatic technique or manual trial and error method.Many thresholding techniques are present to determine the threshold like expectation maximization algorithm[3],ostu ,Kittler and Ilingworth minimum error thresholdingalgorithm.In the case of satellite image change detection is achieved by the accuracy of the classification method and quality of the difference image.To achieve this propose this change detection method. The main two step involved in this techniques are 1)By fusing the mean ratio image and log ratio image ,difference image is produced and 2)To identify the changed and unchanged regions in the difference image, by using the RFLICM clustering technique.This paper is composed of fivesection.Section II involve proposed approach and our motivation will be enhanced.Section III defines the proposed method.In section IV include experimental result and V include conclusion.

\section{Motivaion}

Consider two multi temporal satellite image $\mathrm{x} 1$ and $\mathrm{x} 2$ as the input which is taken from the same geographical area at different time.The motivation is to produce difference image that consist of change information,then only apply image analysis for change detection. According to the fig .1 , proposed change 
detection of image involved in two steps 1)generating the difference image using contourlet fusion and 2) to identify the changed areas in the fused image by reformulated fuzzy clustering.

Due to the multiplicative nature of the speckle noise, the ratio images are b represented in a logarithmic or mean scale. So that good result for the change detection.But this have some disadvantages.In the case of log ratio image, it is not able to reflect the informations of the changed region completely.For the rmd technique, the unchanged regions of the mean ratio images are quite riugh.,and clarity of the image is less.For an optimal image, it should restrain the unchanged areas information and should improve the information of the changed regions. To solve this problem, an image fusion technique is introduced to generate the difference image which is based on the log ratio and mean ratio operation. Sothat the difference image contain better information than individual difference image.Among the fusion methodpixel level image fusion is widely used[4].Discrete wavelet transform is mostly used for pixel level image fusion.It is a multiscale transform technique.But it have a lack of shift invariance property and directional selectivity.For the better change detection one of the important property is the shift invariance property. The image fusion based on the dwt does not preserve the fine edges and curves and the clarity of the image should be very less.So introduce the image fusion based on contourletfusionThe details of this method is described in section III.Themajor purpose is to analyse the difference image is to determine the changed regions and unchanged regions.There are several method is to determine the changed regions like expectation maximization and K\&I algorithm.But these algorithms are mainly used to identify the changed regions. These algorithms are are carried out by applying a thresholding procedure to the histogram of the image.But in addition these methods are require accurate estimation of threshold values.If the estimation is not correct, can not correctly detect the changed region and unchanged regions. So here reformulated fuzzy local information c means clustering algorithm is proposed to analyse the difference image.It is an unsupervisede technique.

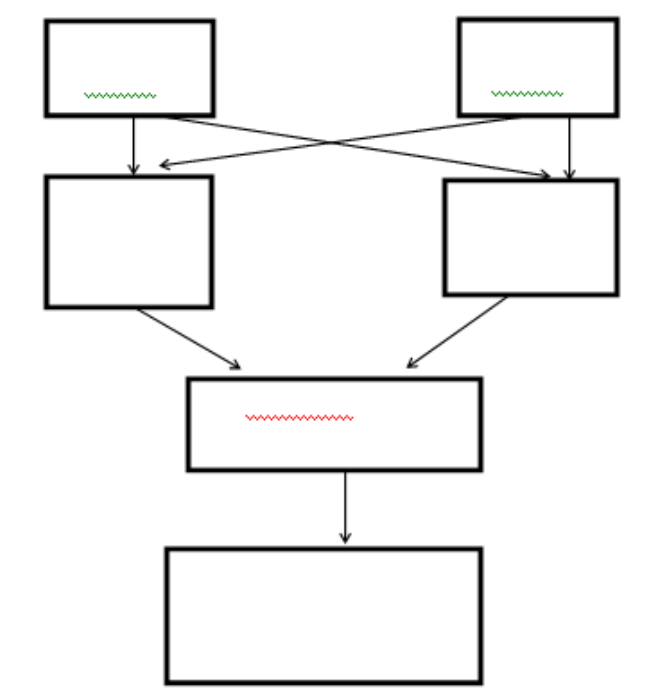

Fig.1. Block diagram of proposed change detection method.

\section{Proposed Methodology}

In this section describes the proposed change detection method,which consist of two steps;1)Generating the difference image using contourlet image fusion,and 2) Detecting changed region using reformulated fuzzy c-means clustering algorithm.

\section{1) Generate the difference image based on contourlet image fusion}

Image fusion is the process of fusing two or more images in to a single image, there by relevant information in the images are combined.So the single image is called diffusev image and which is more informative than the any of the input image[5].All the fusion technique are based on the wavelet transformation.But,the DWT image fusion is resulting with shift variant and additive noise in fused image.It does not preserve edges of the image.So information loss is more.Therefore clarity of the fused image is reduced.These issue can be solved by usingcontourlettransformThe major propertiesofcontourlet transform is ,multiresolution,localization, directionality,anisotropy and local brightness etc.it also provide smoothness to the fused image.This technique is realized by using double iterated filter bank.It uses laplacian pyramid and directional filter bank.There are mainly two steps for implementing this transform. That is transformation and decomposition. 


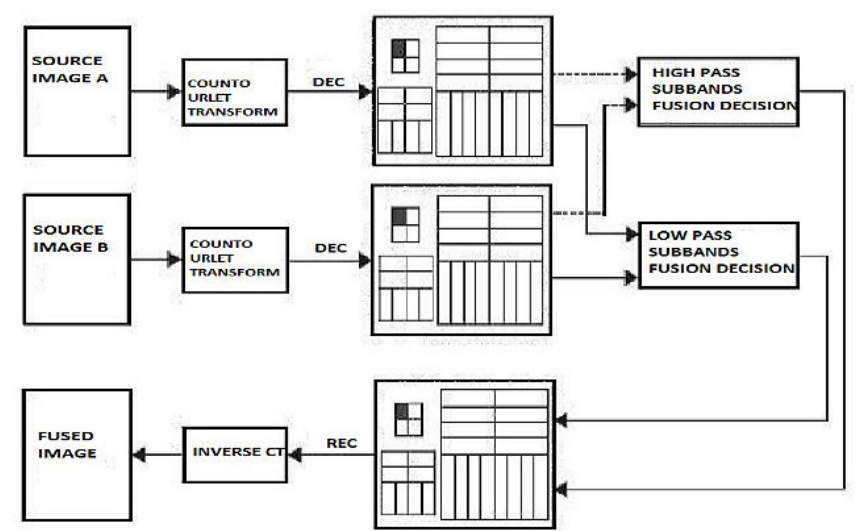

Fig.2. Process of image fusion based on the contourlet.

The two source images used for fusion re obtained from the mean ratio and log ratio operator ,respectively.

$$
\begin{gathered}
X_{m}=1-\min \left(\frac{\mu 1}{\mu 2}, \frac{\mu 2}{\mu 1}\right) \\
X_{l}=\left|\log \frac{X 2}{X 1}\right|=|\log X 2-\log X 1|
\end{gathered}
$$

Where X1 and X2 are multi temporal images and $\mu 1$ and $\mu 2$ present the local mean values of original images.Fig 2 shown above represent the block diagram of the contourlet based image fusion.Here X1 nd X2 denote the input source images respectively.F is the final fused image.The image fusion scheme based on contorlet transform can described as follows.Mainly there are two stages,transformation stage and decomposition stage.

\section{A) Transformation method}

In the transformation stage,for the decomposition of subandsdouble fiter bank is used.It is composed of laplacian pyramid and directional filter bank.So it is called pyramidal directional filter bank.For capturing the edge point,Laplacian pyramid filter is used.Directional Filter Bank is used to link the point discontinuities in the image.

In this method,each input image undergone sub band decomposition. That is in low frequency and bandpasshigh frequency sub band.In the case of low frequency sub band.the same process is repeated up to a specified contourlet decomposition level. The below block diagram fig. 3 show the laplacian pyramid decomposition.

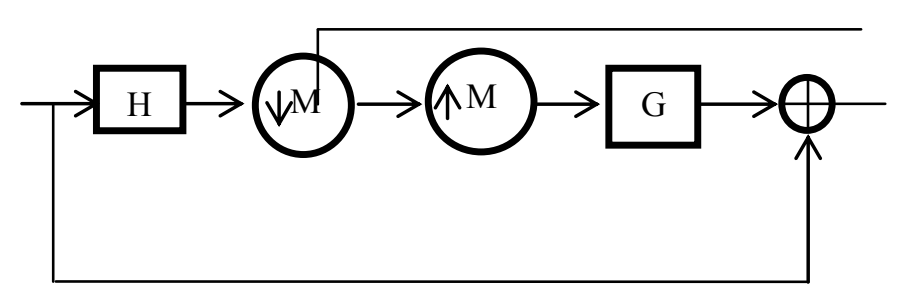

Fig.3. Block diagram of construction of Laplacian pyramid.

Here the input image is fed to the low pass analysis filter $(\mathrm{H})$ and the down sampled to low pass sub band.Then this image is up sampled and applied to the synthesis filter(G).Finally subtracting the output of the synthesis filter and input image getting high pass sub band.Thelaplacian pyramid also allows high frequency bandpass images into further decomposition. That is this bandpass images are passed through the directional filter bank.It captures directional information more accurately.So in this transformation stage, it decomposes the images in to directional subbands at multiscale. 


\section{B)Decomposition method}

In this stage,decomposed sub band of transformation stages are fused by fusion rules. There are separate fusion rule for low pass and high pass band.The coefficient in the low pass sub band represent the profile features of the source image. This is done by comparing each pixel value which is less will be taken as the low pass component.

High frequency fusion rule,described by taking theb mean value as the output.Finally the fused image is obtained from inverse contourlet decomposition method.The proposed method can provide fused image with better visual quality. And also the resultant fused image can preserve much information of edges and textures of satellite image.In the next section describe reformulated fuzzy local information c means clustering algorithm for change detection in contourlet fused image.

\section{2) Analysis of fused image using RFLICM clustering}

Clustering is a process of portioning a data set in to a reasonable number of disjoint groups where each group containing similar samples.In the portioning technique, patterns are similar within the clusters and different between the clusters.In RFLICM the clustering the samples are assigned not only to one cluster,but belong to different clusters. Here clustering is done to discriminate changed regions from unchanged regions .For improving the performance of the image clustering, use the improved version of fuzzy clustering technique. So introduce a fuzzy factor into the object function of RFLICM.The peculiarity of this factor is the use of local similarity measure, which is aimed at ensuring the image detail preservation and noise insensitiveness. The existing analysis higlights the importance of the accurate estimation of the fuzzy factor $G_{k i}$ to suppress effectively the influence of the noisy pixels.But there are some noisy pixel present in the central pixels then we cant remove which also present in the diffused image.In order to over comethis, the local coefficient of variation is adopted .This is defined by

$$
C u=\frac{\operatorname{var}(x)}{(\bar{x})^{2}}
$$

where $\operatorname{var}(x)$, and $\bar{x}$ are the intensity variance and the mean in a local window of the image respectively.

The value of $C_{u}$ reflect the gray value homogeneity degree of the local window .It exhibit high values at the edges or in the area corrupted by noise and produces a low values in homogeneous regions.Compared with the spatial distance ,the discrepancy of the local coefficient of variation between neighbouring pixel and the central pixel is relatively accordance with the gray level difference between them.In addition it helps to exploit more local context information since the local coefficient of variation of variation of each pixel is computed in a local window.Here the modified fuzzy factor $G_{k i}^{\prime}$ can be defined as

$$
\begin{gathered}
G_{k i}^{\prime}= \\
\left\{\begin{array}{l}
\sum_{j \in N_{i}} \frac{1}{2+\min \left(\left(\frac{c_{u}^{j}}{c_{u}}\right)^{2},\left(\frac{c_{u}}{c_{u}^{j}}\right)^{2}\right)} \times\left(1-u_{k j}\right)^{m}\left\|x_{j}-v_{k}\right\|^{2}, \text { if } C_{u}^{j} \geq \overline{C_{u}} \\
\sum_{j \in N_{i}} \frac{1}{2-\min \left(\left(\frac{c_{u}^{j}}{C_{u}}\right)^{2},\left(\frac{c_{u}}{c_{u}^{j}}\right)^{2}\right)} \times\left(1-u_{k j}\right)^{m}\left\|x_{j}-v_{k}\right\|^{2}, \text { if } C_{u}^{j} \overline{\leq C_{u}}
\end{array}\right.
\end{gathered}
$$

Where $C_{u}$ s the local coefficient of variation of the central pixel, $C_{u}^{j}$ represent the local coefficient of variation of neighbouringpixel, and $\overline{C_{u}}$ is the mean value of $C_{u}^{j}$ that is located in a local window. As shown in (4), the reformulated factor $G_{k i}^{\prime}$ balances the membership value of the central pixel taking in to account the local coefficient of variation ,as well as the gray level of theneighbouringpixel.If there is distinct difference between the result of the local coefficient of variartions that are obtained by the neighbouring pixel and the central pixel,the weightings added of the neighbouring pixel in $G_{k i}^{\prime}$ will be increased to suppresses the influence of outlier; Therefor the RFLICM to be more robust .

By using the definition of $G_{k i}^{\prime}$ the objective function of the RFLICM can be defined in terms of

$$
J(m)=\sum_{i=1}^{N} \sum_{k=1}^{c}\left[U_{i j}^{m}\|x i-v k\|^{2}+G_{k i}^{\prime}\right], 1 \leq \infty(5)
$$


Where $v_{k}$ represent the prototype value of the $k$ th cluster and $U_{i j}$ represent fuzzy membership of the $i$ th pixel with resepect to cluster $k, N$ is the number of data items, and $c$ is the number of clusters. $\left\|x_{i}-v_{k}\right\|$ is the Euclidian distance between object $x_{i}$ and the cluster centre $v_{k}$.

In addition ,the calculation of the membership partition matrix and the cluster centersis performed as follows:

$U_{i j}=\frac{1}{\sum_{k=1}^{C}\left(\frac{\|x i-c j\|}{\|x i-c k\|}\right)^{2 / m-1}}$

$C_{j}=\frac{\sum_{i=1}^{N} U_{i j}^{m} x i}{\sum_{i=1}^{N} U_{i j}^{m}}$

Where the membership partition matrix is computed randomly.By taking the fuzzy factor $G_{k i}^{\prime}$ the RFLICM algorithm can be summarized as follows:

1) Set values of $c, m$, and $\in$.

2) Initialize randomly the fuzzy partition matrix and set the loop counter $b=0$.

3) Calculate the cluster prototype using (7).

4) Compute the partition matrix using (6).

5) $\max \left\{U^{(b)}-U^{(b+1)}\right\}<\in$ then stop ;otherwise, set $b=b+1$,and go to step 3).

\section{Result And Discussion}

The change detection of satellite image based on the image fusion and reformulated fuzzy c means clustering algorithm. The changed and unchanged areas are correctly classified .In order to analyse percetage of correct classification is taken.

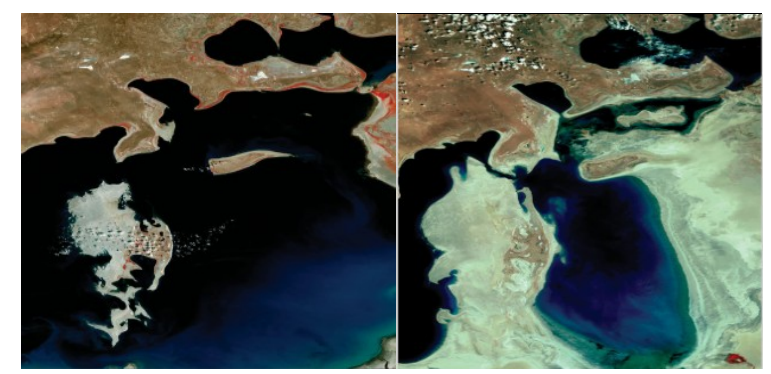

(a)

(b)

Fig.6. Two input image. (a) Image 1. (b )Image2.

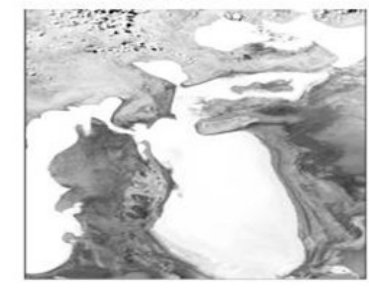

(a)

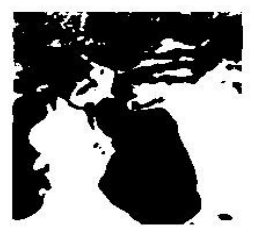

(b)

Fig.7. fusion and change detection. (a) Fused image. (b) Clustered image

A comparative analysis for the suitability of the proposed approach for the fused difference image.For the quantitative analysis of change detection, calculate the percentage correct classification by

$$
\mathrm{PCC}=(\mathrm{TP}+\mathrm{TN}) /(\mathrm{TP}+\mathrm{FP}+\mathrm{TN}+\mathrm{FN})(6)
$$

Here,TP is the number of pixels that are detected as the changed areas.TN is the number of pixels that are detected as the unchanged areas.The false negative (FN)are the changed pixels that an undetected.False positive(FP) is the unchanged pixels wrongly classified as changed.This percentage of correct classification will give the correctness of our approach.The changed areas should enhance and un cahnaged areas should retain. The changed areas should representing by a binary equal to one and unchanged areas should representing 
by a binary of zero.Because there are two classes of clustering. One is representing by one and other by zero.So that we get ba correct classification.

Table1

Change detection results based on contourlet image fusion

\begin{tabular}{|c|c|c|c|}
\hline Difference image & FP & FN & PCC \\
\hline Mean Ratio & 3195 & 12302 & 56.5484 \\
\hline Log Ratio & 11499 & 7329 & 47.2087 \\
\hline Contourlet fusion & 385 & 7244 & 78.6093 \\
\hline
\end{tabular}

\section{Conclusion}

Represented the unsupervised approach based on contourlet image fusion and RFLICM clustering for change detection in satellite images.In order to restrain the unchanged areas and enhance the changed areas ,fusion approach is used.Among the fusion method,the limitations of the wavelet transform is capturing the geometry of the image edges. So pursue contourlet transform that can capture the intrinsic geometrical structure that is key in visual information.In this method can provide a fused image with better quality.In addition to that difference image produced in this method is better than that of dwt fused difference image. The obtained fused image will be analyzing by RFLICM clustering for the change detection. So which give a better performance than the pre existed one.

\section{References}

[1] MaoguoGong,MemberIEEE,zhiqiangzhou,andjingjingMa, "Change detection in synthetic aperture radar based on image fusion and fuzzy clustering," April 2012.

[2] MerinAyshuAli,DR.B.M.Imran, "Change detection in SAR image using contourlet," International journal of scientific and engineering research volume 4,August 2013

[3] PalleE.T.Jorgensen and Myung-sin song, "Comparison of discrete and continuos wavelet transforms," , Mar. 2010

[4] Yakoubbazi,Lorenzobruzzone,faridmelgani, "Image thresholding based on the EM algorithm and the generalized gaussian distribution,"epartment of information and communication technologies, university of Trento,via sommarive,14,I38050,Trento,italy, May. 2006.

[5] C.K Leung and F.K Lam, "Performance analysis for aclass of iterative imagethresholding algorithms ," Department of electronics engineering,Thehongkongpolytechinicuniversity,hong kong,Depratment of electrical and electronics engineering,,the university ofhongkong,hongkong, january. 1996..

[7] Gabriele moser, "Generalized minimum error thresholding for unsupervised change detection from SAR amplitude imagery," member IEEE, andsebastianoB.serpico,senior member ,IEEE Tranaction geosceience remote sensing, vol,44,no.10,October 2006.

[8] Uzaykaymak and magnesetnes, “Extended fuzzy clustering algorithm," IEEETrans., Oct.2009. 